\title{
Regiochemistry in Pd-Catalysed Organotin Reactions with Halopyrimidines
}

\author{
Jan Solberg and Kjell Undheim \\ Department of Chemistry, University of Oslo, 0315 Oslo 3, Norway
}

\begin{abstract}
Solberg, J. and Undheim, K., 1989. Regiochemistry in Pd-Catalysed Organotin Reactions with Halopyrimidines. - Acta Chem. Scand. 43: 62-68.

Chlorines in activated pyrimidine position is replaced by carbon substituents in Pd-catalysed reactions with organotin compounds. The 4(6)-position is more reactive than the 2-position allowing for regioselective coupling in 2,4(6)-dihalopyrimidines. A bromine substituent is required for coupling in the benzenoid 5-position. In 5-bromo-2,4-dichloropyrimidine the 4-chlorine is replaced before the 5-bromine and the latter before the 2-chloro substituent, all in a regioselective manner. The methodology can be used to introduce functionalized carbon substituents into any pyrimidine position.
\end{abstract}

From our efforts to develop methodology for the introduction of carbon substituents into the pyrimidine ring we have reported that this can be achieved by adduct formation between the $\pi$-electron deficient pyrimidine ring and organometallic reagents and subsequent rearomatization, ${ }^{1}$ by Pd-catalysed cross-coupling reactions using 4-iodopyrimidines, ${ }^{2,3}$ and by reactions of 5-pyrimidinyltin derivatives. ${ }^{4}$ In this report we describe a general study of coupling reactions in chloropyrimidines. The latter, often in contrast with the corresponding iodopyrimidines, are readily available substrates e.g. from reactions between hydroxypyrimidines and phosphorus chlorides. ${ }^{5}$ The coupling reactions were achieved using organotin reagents and Pd catalysis.
In Scheme 1 it is shown that 2-chloropyrimidines couple readily with the phenyl or styryl group in tributyltin reagents in the presence of dichlorobis(triphenylphosphine)palladium(II). The solvent was dimethylformamide (DMF). The reaction temperatures, times and yields are tabulated in the scheme.

Coupling occurs exclusively in the activated 2-position in the 2,5-dichloro derivative $\mathbf{1 b}$ because the 5-position in pyrimidines is benzenoid and by analogy to arenes generally must carry a bromo or iodo substituent for coupling to occur. ${ }^{6}$ In 5-bromo-2-chloropyrimidine (1c) coupling could, in principle, occur in either position, but exclusive formation of the mono-coupled product 5 by replacement

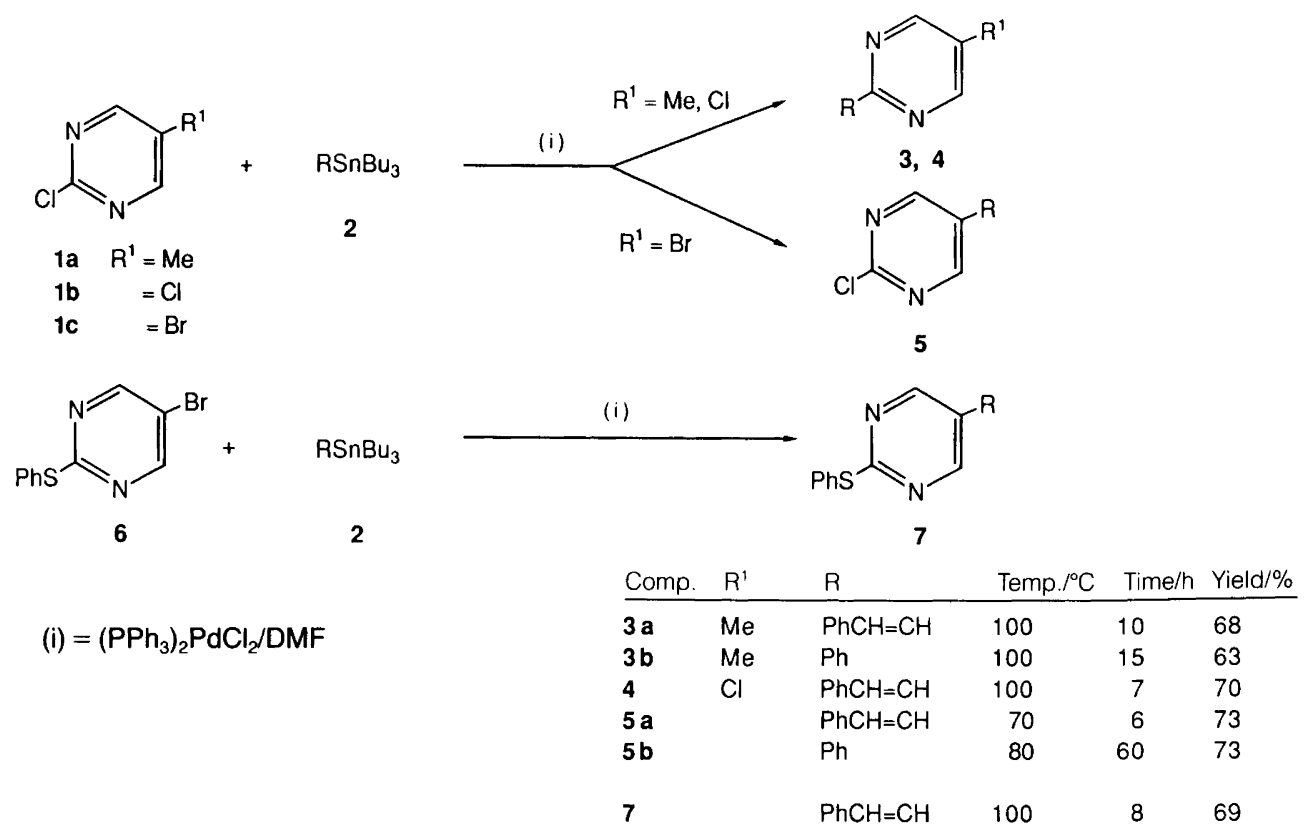

Scheme 1.

62 Acta Chemica Scandinavica (1989) 62-68 


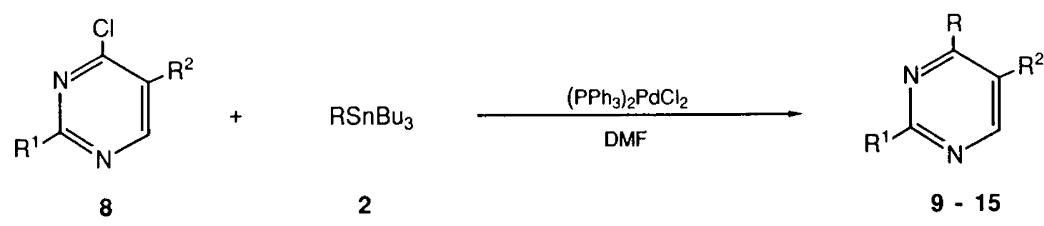

$\begin{array}{lll}\text { Comp } & \mathrm{R}^{1} & \mathrm{R}^{2} \\ \mathbf{8 a} & \mathrm{MeS} & \mathrm{H} \\ \mathbf{8 b} & \mathrm{MeSO}_{2} & \mathrm{H} \\ & & \\ \mathbf{8 c} & \mathrm{MeS} & \mathrm{Cl} \\ \mathbf{8 d} & \mathrm{Cl} & \mathrm{H} \\ \mathbf{8 e} & \mathrm{Cl} & \mathrm{Cl} \\ \mathbf{8 f} & \mathrm{Cl} & \mathrm{Br} \\ \mathbf{8 g} & \mathrm{MeS} & \mathrm{Br}\end{array}$

\begin{tabular}{|c|c|c|c|c|c|c|}
\hline Comp. & $\mathrm{R}^{1}$ & $\mathrm{R}^{2}$ & $\mathrm{R}$ & Temp. $/{ }^{\circ} \mathrm{C}$ & Time/h & Yield $/ \%$ \\
\hline $9 a$ & $\mathrm{MeS}$ & $H$ & $\mathrm{PhCH}=\mathrm{CH}$ & 100 & 1.3 & 85 \\
\hline $\begin{array}{r}9 b \\
10 a\end{array}$ & $\begin{array}{l}\mathrm{MeS} \\
\mathrm{MeSO}_{2}\end{array}$ & $\stackrel{\mathrm{H}}{\mathrm{H}}$ & $\begin{array}{l}\mathrm{Ph} \\
\mathrm{PhCH}=\mathrm{CH}\end{array}$ & $\begin{array}{r}100 \\
80\end{array}$ & $\begin{array}{l}8 \\
2\end{array}$ & $\begin{array}{l}60 \\
65\end{array}$ \\
\hline $10 \mathrm{~b}$ & $\mathrm{MeSO}_{2}$ & $\mathrm{H}$ & $\mathrm{Ph}$ & 100 & 2.5 & 65 \\
\hline $10 \mathrm{c}$ & $\mathrm{MeSO}_{2}$ & $\mathrm{H}$ & $\mathrm{Bu}$ & 100 & 5.5 & 56 \\
\hline $11 \mathrm{a}$ & MeS & $\mathrm{Cl}$ & $\mathrm{PhCH}=\mathrm{CH}$ & 100 & 3.5 & 66 \\
\hline $11 \mathrm{~b}$ & MeS & $\mathrm{Cl}$ & $\mathrm{Ph}$ & 100 & 5 & 66 \\
\hline $12 a$ & $\mathrm{Cl}$ & $\mathrm{H}$ & $\mathrm{PhCH}=\mathrm{CH}$ & 70 & 7 & 77 \\
\hline $12 b$ & $\mathrm{Cl}$ & $H$ & $\mathrm{Ph}$ & 80 & 10 & 60 \\
\hline $13 a$ & $\mathrm{Cl}$ & $\mathrm{Cl}$ & $\mathrm{PhCH}=\mathrm{CH}$ & 70 & 3 & 71 \\
\hline $13 b$ & $\mathrm{Cl}$ & $\mathrm{Cl}$ & $\mathrm{Ph}$ & 80 & 10 & 69 \\
\hline 14 & $\mathrm{Cl}$ & $\mathrm{Br}$ & $\mathrm{PhCH}=\mathrm{CH}$ & 70 & 6 & 73 \\
\hline 15 & MeS & $\mathrm{Br}$ & $\mathrm{PhCH}=\mathrm{CH}$ & 50 & 27 & 66 \\
\hline
\end{tabular}

Scheme 2.

of the bromine was observed. 5-Bromo-2-phenylthiopyrimidine (6) under similar reaction conditions gave the product 7 .

Scheme 2 deals with 4-chloropyrimidines which are shown to couple readily with organotin reagents. The reactivities follow the established order for organotin compounds; $\mathrm{R}=\mathrm{PhCH}=\mathrm{CH}>\mathrm{Ph} \gg \mathrm{Bu} .{ }^{6}$ Replacement of the 2-sulfide group in $\mathbf{8 a}$ with the strongly electron-withdrawing sulfonyl group in $\mathbf{8 b}$ increases the ease of coupling in the chloro substituted 4-position. This finding is in good agreement with literature reports that electron-withdrawing groups accelerate coupling reactions, and that electronwithdrawing groups are required for chlorobenzenes to react. $^{7}$

In the case of the sulfonyl derivative $\mathbf{8 b}$, a butyl group could be introduced to furnish 10c. In most cases, however, vigorous conditions were necessary to effect the alkylation resulting in heterogeneous products.

In the 4,5-dichloro derivative $8 \mathrm{c}$ coupling occurs in the activated 4-position. In 2,4-dichloropyrimidine (8d), in which both chlorine positions are activated, exclusive coupling in the 4-position was observed. The latter is also the more reactive position in nucleophilic substitution reactions. Based on the above findings, it was no surprise to find that regioselective coupling can be achieved in the 4-position in 2,4,5-trichloropyrimidine (8e). It is, however, noteworthy that in the 5-bromo analogue 8f, the first coupling takes place in the chloro substituted 4-position and not in the bromo substituted 5-position, the product being compound 14. The 2-methylthio analogue $8 \mathrm{~g}$ reacts in the same manner with substitution at $\mathrm{C}-4$.

The difference in reactivity between the 4-chloro and 5-bromo substituents in $\mathbf{8 g}$ and $\mathbf{8 f}$ is relatively small and regioselectivity was not achieved using the phenyltin reagent. Using the more reactive styryltin reagent and milder reaction conditions, however, regioselectivity can be achieved. For comparison, in 5-bromo-2-chloropyrimidine (1c) and 2,4-dichloropyrimidine (8d) the difference in reactivity between the halogens is larger, allowing for regioselective phenylation.<smiles>[X]c1cnc([X]([Y])(Br)Br)nc1/C=C\c1ccccc1</smiles>

15
$16 \quad X=C l$
$17 X=B r$
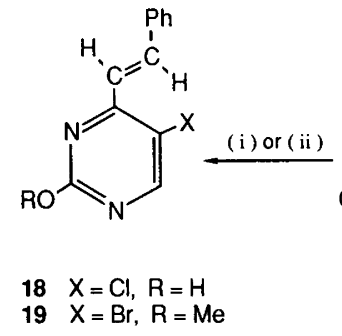<smiles>[X]c1cnc(Cl)nc1/C=C/c1ccccc1</smiles>

$18 X=\mathrm{Cl}, \mathrm{R}=\mathrm{H}$

$19 \mathrm{X}=\mathrm{Br}, \mathrm{R}=\mathrm{Me}$
$13 a$
14

(i) $1 \mathrm{M} \mathrm{NaOh} /$ Dioxane $/ \Delta$

Scheme 3.

(ii) $\mathrm{NaOMe} / \mathrm{MeOH} / \Delta$ 


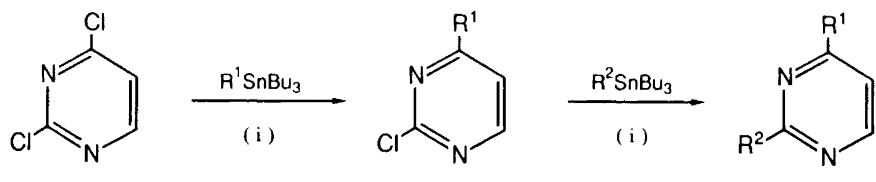<smiles>Clc1ncc(Br)c(Cl)n1</smiles>

\begin{tabular}{lllccc} 
Comp. & $\mathrm{R}^{1}$ & $\mathrm{R}^{2}$ & Temp.${ }^{\circ} \mathrm{C}$ & Time/h & Yield $/ \%$ \\
\hline 20a & $\mathrm{PhCH}=\mathrm{CH}$ & $\mathrm{Ph}$ & 130 & 15 & $54^{\mathrm{a}}$ \\
20b & $\mathrm{Ph}$ & $\mathrm{PhCH}=\mathrm{CH}$ & 100 & 10 & $68^{\mathrm{a}}$ \\
21 & & & 80 & 50 & $70^{\mathrm{b}}$ \\
22 & & & 100 & 15 & $82^{\mathrm{c}}$
\end{tabular}

The regiochemistry of the reactions between 2,4,5-trichloropyrimidine (8e) and its 5-bromo analogue $8 \mathrm{f}$ with the styryltin reagent was verified by separate reactions (Scheme 3). The products 13a and 14 were treated with sodium hydroxide in aqueous dioxane or sodium methoxide in methanol to furnish the hydroxy or methoxy compounds 18 and 19, respectively. The same compounds were available from the corresponding 2-methylthio-4-styrylpyrimidines $11 \mathrm{a}$ and 15 which were oxidized by $m$-chloroperbenzoic acid to the sulfones $\mathbf{1 6}$ and $\mathbf{1 7}$ and solvolysed.

The tributyl- $\beta$-styryltin reagent was a cis/trans mixture, ratio 13:87. The crude product from the coupling reactions had the same stereochemical composition in agreement with literature reports on the coupling between vinyltin reagents and aryl halides. ${ }^{8}$ On purification, however, the trans isomer was isolated and the physical data recorded in the experimental section are those for the trans isomers.

Regioselective coupling at C-4 in 2,4-dichloropyrimidine (8d) yields compounds 12 (Scheme 4). The second chlorine in the product 12 at $\mathrm{C}-2$ can be replaced by a new coupling reaction as seen in the formation of the carbon disubstituted pyrimidines 20. Previously it has been reported that regioselectivity could not be achieved in coupling reactions of 2,4-diiodo-6-methylpyrimidine or the isomeric 4,6-diiodo-2-methylpyrimidine with terminal alkynes using dichlorobis(triphenylphosphine)palladium(II) catalysis in the presence of $\mathrm{Cu}(\mathrm{I})$ iodide. ${ }^{9}$ But our findings with the organotin reagents parallel reactions recently reported between thiopheneboronic acids and 2,4-dibromo- and 2,4-dichloropyrimidines in which regioselective substitution of the halogen in the 4-position was observed using tetrakis(triphenylphosphine)palladium $(0)$ as the catalyst. ${ }^{10}$

5-Bromo-2,4-dichloropyrimidine (8f) was used to demonstrate the stepwise introduction of three different carbon substituents (Scheme 4). Compound 8 f reacted initially with replacement of the chlorine at C-4 using tributyl- $\beta$ styryltin. Treatment of the product 14 with the phenyltin reagent brought about phenylation at C-5 to give 21 . The halogen remaining in the 2-position was replaced by a thienyl group (compound 22) when the former was treated with tributyl(2-thienyl)tin.

Functionalized carbon substituents can be introduced into both activated and non-activated positions in halopyrimidines. Thus for the preparation of the ketones 24 (Scheme 5) the starting material was ethyl vinyl ether which was lithiated at the $\alpha$-vinyl carbon and treated with tributyltin chloride. The stannylated vinyl ether can be isolated and purified by distillation. This then reacts readily with halopyrimidines to yield the ethoxyvinylpyrimidines $\mathbf{2 3}$ which, on mild acid hydrolysis, give the corresponding ketones 24. This reaction sequence constitutes a convenient method for the introduction of an acyl function into any pyrimidine position.

\section{Experimental}

The ${ }^{1} \mathrm{H}$ and the ${ }^{13} \mathrm{C}$ NMR spectra were recorded in $\mathrm{CDCl}_{3}$ at 60 and $75 \mathrm{MHz}$ respectively, unless otherwise specified. The mass spectra were recorded under electron impact conditions at $70 \mathrm{eV}$ ionizing voltage.

Starting materials available by literature methods. Tributyl- $\beta$-styryltin, ${ }^{11}$ tributyl-(2-thienyl)tin, ${ }^{12}$ tributyl(phenyl) tin, ${ }^{13}$ 2-chloro-5-methylpyrimidine (1a), ${ }^{14}$ 2,-dichloropyrimidine (1b), ${ }^{15}$ 5-bromo-2-chloropyrimidine (1c), ${ }^{16}$ 5bromo-2-phenylthiopyrimidine (6), ${ }^{17}$ 4-chloro-2-methylthiopyrimidine (8a), ${ }^{18}$ 4-chloro-2-methylsulfonylpyrimidine (8b), ${ }^{19}$ 4,5-dichloro-2-methylthiopyrimidine (8c) ${ }^{2}{ }^{2}$ 2,4-di chloropyrimidine (8d), ${ }^{10}$ 2,4,5-trichloropyrimidine $(\mathbf{8 e}),{ }^{20}$ 

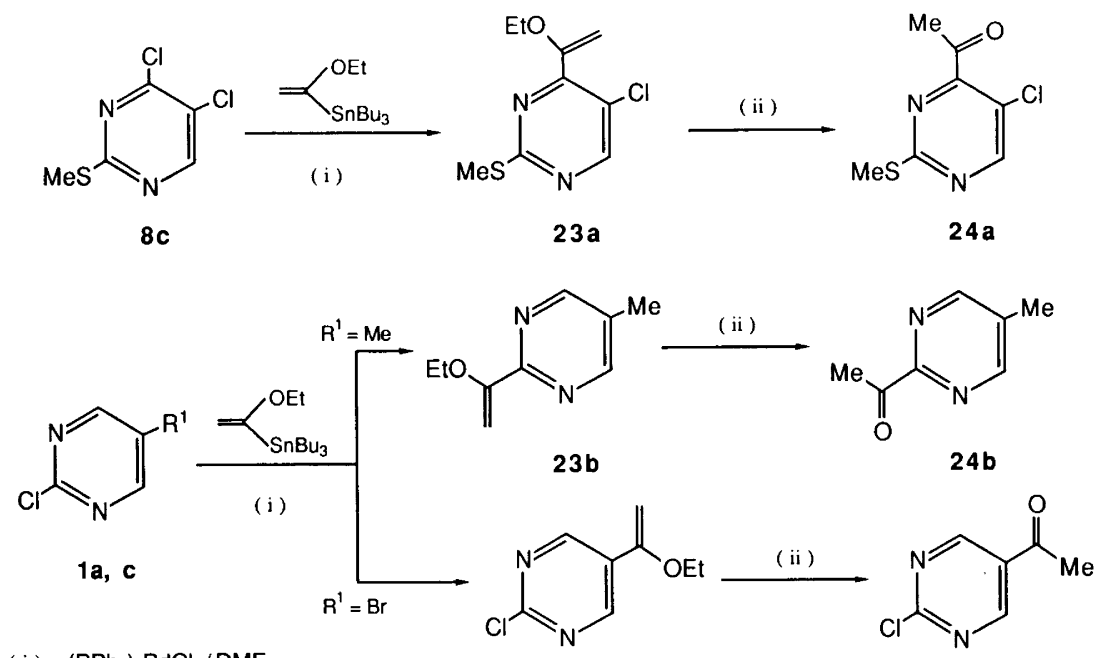

(i) $\left(\mathrm{PPh}_{3}\right)_{2} \mathrm{PdCl}_{2} / \mathrm{DMF}$

(ii) $1 \mathrm{M} \mathrm{HCl} /$ acetone, $1: 4$

Scheme 5.

5-bromo-2.4-dichloropyrimidine (8f), ${ }^{21}$ 5-bromo-4-chloro2-methylthiopyrimidine $(\mathbf{8 g}),{ }^{22}$ and tributyl- $\alpha$-ethoxyethenyltin. ${ }^{23}$

General procedure for Pd-catalysed coupling reactions between halopyrimidines and organotin compounds. Dichlorobis(triphenylphosphine)palladium(II) $\quad(70 \mathrm{mg}$, $0.10 \mathrm{mmol}$ ) was added to a solution of the halopyrimidine $(5.00 \mathrm{mmol})$ and the organotin compound $(5.50 \mathrm{mmol})$ in dry DMF $(10 \mathrm{ml})$. The mixture was heated in a $\mathrm{N}_{2}$ atmosphere at the temperatures and for the time given in the schemes. The progress of the reactions was monitored by GLC or TLC. A concentrated aqueous solution of potassium fluoride $(20 \mathrm{ml})$ was added to the cold reaction mixture followed by diethyl ether $(30 \mathrm{ml})$. The mixture was stirred vigorously for $30 \mathrm{~min}$, after which time water (100 $\mathrm{ml}$ ) was added and the mixture was extracted with diethyl ether $(3 \times 50 \mathrm{ml})$. The ether extracts were combined, washed with water $(3 \times 50 \mathrm{ml})$, dried $\left(\mathrm{MgSO}_{4}\right)$, and evaporated, and the product was purified by flash chromatography on silica gel and finally recrystallized or subjected to Kugelrohr distillation.

5-Methyl-2-( $\beta$-styryl)pyrimidine (3a). ${ }^{24}$ DichloromethaneEtOAc 99:1 was used for flash chromatography; m.p. $141^{\circ} \mathrm{C}$ (EtOH). Anal $\mathrm{C}_{13} \mathrm{H}_{12} \mathrm{~N}_{2}: \mathrm{C}, \mathrm{H} .{ }^{1} \mathrm{H}$ NMR (300 MHz): $\delta 2.26(\mathrm{Me}), 7.22$ and $7.92(\mathrm{CH}=\mathrm{CH}, 2 \times \mathrm{d}, J 16.0 \mathrm{~Hz})$, 7.34 and $7.60(\mathrm{Ph}, 2 \times \mathrm{m}), 8.52(\mathrm{H}-4,6) .{ }^{13} \mathrm{C}$ NMR: $\delta 15.3$ $(\mathrm{Me}), 127.2$ and $128.6\left(\mathrm{CH}=\right.$ and $\left.\mathrm{C}_{\mathrm{p}}\right), 127.3$ and $128.5\left(\mathrm{C}_{o}\right.$ and $\left.\mathrm{C}_{m}\right), 127.5(\mathrm{C}-5), 136.0\left(\mathrm{C}_{\mathrm{i}}\right), 136.7(\mathrm{CH}=), 156.9(\mathrm{C}-4$, 6), 162.3 (C-2). MS: $196(27, M), 195(100), 181$ (1), 168 (2), 167 (1), 129 (6), 128 (6).
5-Methyl-2-phenylpyrimidine (3b). ${ }^{25}$ Dichloromethane was used for flash chromatography; m.p. $69^{\circ} \mathrm{C}(\mathrm{EtOH})$. Anal. $\mathrm{C}_{11} \mathrm{H}_{10} \mathrm{~N}_{2}$ : C, H. ${ }^{1} \mathrm{H}$ NMR: $\delta 2.26(\mathrm{Me}), 7.5$ and $8.4(\mathrm{Ph}, 2$ $\times \mathrm{m}), 8.62(2 \mathrm{H}, \mathrm{s}, \mathrm{H}-4,6)$. MS: $170(87, M), 144(2), 143$ (15), $116(2), 115$ (5), 104 (21), 103 (100).

5-Chloro-2-( $\beta$-styryl)pyrimidine (4). Toluene was used for flash chromatography; m.p. $131^{\circ} \mathrm{C} \quad(\mathrm{EtOH})$. Anal. $\mathrm{C}_{12} \mathrm{H}_{9} \mathrm{ClN}_{2}$ : C, H. ${ }^{1} \mathrm{H}$ NMR: $\delta 7.20$ and $8.00(\mathrm{CH}=\mathrm{CH}, 2 \times$ d, J $16 \mathrm{~Hz}) 7.2-7.8(\mathrm{Ph}), 8.65(2 \mathrm{H}, \mathrm{s}, \mathrm{H}-4,6)$. MS: $218 / 216$ (7/25, M), 217/215 (32/100), 181 (3), 154 (3), 153 (2), 152 (2), 129 (11), $128(8)$.

2-Chloro-5-( $\beta$-styryl)pyrimidine (5a). Dichloromethane was used for flash chromatography; m.p. $146^{\circ} \mathrm{C}(\mathrm{EtOH})$. Anal. $\mathrm{C}_{12} \mathrm{H}_{9} \mathrm{ClN}_{2}$ : C, H. ${ }^{1} \mathrm{H}$ NMR: $\delta \quad 6.85$ and 7.12 $(\mathrm{CH}=\mathrm{CH}, 2 \times \mathrm{d}, \mathrm{J} 17 \mathrm{~Hz}), 7.2-7.5(\mathrm{Ph}), 8.61(2 \mathrm{H}, \mathrm{s}, \mathrm{H}-4$, 6). MS: $218 / 216(29 / 91, M), 217 / 215$ (42/100), 201 (2), 190 (2), 189 (3), 188 (5), 182 (3), 181 (28), 180 (8), 179 (52).

2-Chloro-5-phenylpyrimidine (5b). ${ }^{26}$ Dichloromethane was used for flash chromatography; m.p. $132^{\circ} \mathrm{C}(\mathrm{EtOH})$. Anal. $\mathrm{C}_{10} \mathrm{H}_{7} \mathrm{ClN}_{2}$ : C, H. ${ }^{1} \mathrm{H}$ NMR (300 MHz): $\delta$ 7.48-7.80 (Ph), $8.82(\mathrm{H}-4,6) .{ }^{13} \mathrm{C}$ NMR: $\delta 126.8$ and $129.4\left(\mathrm{C}_{o}\right.$ and $\left.\mathrm{C}_{m}\right)$, $129.2\left(\mathrm{C}_{p}\right), 132.8$ and $132.9\left(\mathrm{C}-5\right.$ and $\left.\mathrm{C}_{\mathrm{i}}\right), 157.3(\mathrm{C}-4,6)$, 160.1 (C-2). MS: 192/190 (32/100, M), 191 (12), 189 (3), $156(2), 155$ (16), 129 (2), 128 (8), 102 (55).

2-Phenylthio-5-( $\beta$-styryl)pyrimidine (7). Toluene-EtOAc 99:1 was used for flash chromatography; m.p. $109^{\circ} \mathrm{C}$ (EtOH). Anal. $\mathrm{C}_{18} \mathrm{H}_{14} \mathrm{~N}_{2} \mathrm{~S}: \mathrm{C}, \mathrm{H} .{ }^{1} \mathrm{H}$ NMR (300 MHz): $\delta$ 6.88 and $7.10(\mathrm{CH}=\mathrm{CH}, 2 \times \mathrm{d}, J 16.4 \mathrm{~Hz}), 7.2-7.7(2 \times$ 
Ph), 8.61 (2 H, s, H-4, 6) MS: $290(100, M), 289$ (36), 284 (2), 283 (5), 233 (17), 232 (91).

2-Methylthio-4-phenylpyrimidine (9b). ${ }^{27}$ Toluene-EtOAc ane-light petroleum 3:1 was used for flash chromatography. The analytical samlple was prepared by Kugelrohr distillation, b.p. $160^{\circ} \mathrm{C} / 0.02 \mathrm{mmHg}$. Anal. $\mathrm{C}_{13} \mathrm{H}_{12} \mathrm{~N}_{2} \mathrm{~S}$ : C, H. ${ }^{1} \mathrm{H}$ NMR (300 MHz): $\delta 2.60(\mathrm{MeS}), 6.91(1 \mathrm{H}, \mathrm{d}, J 5.2$ $\mathrm{Hz}, \mathrm{H}-5), 6.95$ and $7.84(\mathrm{CH}=\mathrm{CH}, 2 \times \mathrm{d}, J 16.0 \mathrm{~Hz}), 7.3$ and $7.6(\mathrm{Ph}, 2 \times \mathrm{m}), 8.42(1 \mathrm{H}, \mathrm{d}, J 5.1 \mathrm{~Hz}, \mathrm{H}-6)$. MS: 228 (100, M), 227 (46), 213 (4), 195 (9), 182 (17), 181 (46), 155 (19), 154 (14).

2-Methylthio-4-phenylpyrimidine (9b). ${ }^{27}$ Toluene-EtOAc $98: 2$ was used for flash chromatography; m.p. $88^{\circ} \mathrm{C}$ (EtOH). Mol.wt: Found 202.0574. Calc. for $\mathrm{C}_{11} \mathrm{H}_{10} \mathrm{~N}_{2} \mathrm{~S}$ : 202.0565. 'H NMR: $\delta 2.67$ (SMe), $7.35(1 \mathrm{H}, \mathrm{d}, J 5 \mathrm{~Hz}$, $\mathrm{H}-5), 7.5$ and $8.1(\mathrm{Ph}, 2 \times \mathrm{m}), 8.53(1 \mathrm{H}, \mathrm{d}, J 5 \mathrm{~Hz}, \mathrm{H}-6)$. MS: $202(100, M), 201(32), 157$ (8), $156(46), 155(29), 129$ (17), $102(11)$.

2-Methylsulfonyl-4-( $\beta$-styryl)pyrimidine (10a). The crude product was washed with light petroleum and recrystallized from ethanol, m.p. $115^{\circ} \mathrm{C}$. Anal. $\mathrm{C}_{13} \mathrm{H}_{12} \mathrm{~N}_{2} \mathrm{O}_{2} \mathrm{~S}$ : C, H. ${ }^{1} \mathrm{H}$ NMR: $\delta 3.35\left(\mathrm{MeSO}_{2}\right), 7.05$ and $7.98(\mathrm{CH}=\mathrm{CH}, 2 \times \mathrm{d}, J$ $16 \mathrm{~Hz}$ ), 7.2-7.7 (Ph and H-5), $8.73(1 \mathrm{H}, \mathrm{d}, J 5 \mathrm{~Hz}, \mathrm{H}-6)$. MS: $260(11, M), 259(17), 197(8), 182(6), 181(48), 180$ (100), 154 (24), 153 (16).

2-Methylsulfonyl-4-phenylpyrimidine (10b). ${ }^{27}$ The crude product was washed with light petroleum and recrystallized from ethanol, m.p. $136^{\circ} \mathrm{C}$. Anal. $\mathrm{C}_{11} \mathrm{H}_{10} \mathrm{~N}_{2} \mathrm{O}_{2} \mathrm{~S}: \mathrm{C}, \mathrm{H} .{ }^{1} \mathrm{H}$ NMR: $\delta 3.42\left(\mathrm{MeSO}_{2}\right), 7.5$ and $8.0(\mathrm{Ph}, 2 \times \mathrm{m}), 7.90(1 \mathrm{H}$, d, J $6 \mathrm{~Hz}, \mathrm{H}-5), 8.85$ (1 H, d, J $6.0 \mathrm{~Hz}, \mathrm{H}-6)$. MS: 234 (30, M), 219 (10), 271 (16), 156 (20), 155 (100), 103 (30).

4-Butyl-2-methylsulfonylpyrimidine (10c). EtOAc-hexane 1:1 was used for flash chromatography. The analytical sample was prepared by Kugelrohr distillation, b.p. $150^{\circ} \mathrm{C} / 0.005 \mathrm{mmHg}$. Anal. $\mathrm{C}_{9} \mathrm{H}_{14} \mathrm{~N}_{2} \mathrm{O}_{2} \mathrm{~S}: \mathrm{C}, \mathrm{H} .{ }^{1} \mathrm{H}$ NMR: $\delta$ 1.1-2.9 (Bu), $3.35\left(\mathrm{MeSO}_{2}\right), 7.46(1 \mathrm{H}, \mathrm{d}, J 5 \mathrm{~Hz}, \mathrm{H}-5)$, 8.78 (1 H, d, J 5 Hz, H-6). MS: $214(0.3, M), 199$ (3), 186 (3), 185 (23), 174 (5), 173 (7), 172 (100), 135 (8).

5-Chloro-2-methylthio-4-( $\beta$-styryl)pyrimidine (11a). ${ }^{3}$ The crude product was purified by recrystallization from ethanol, m.p. $81^{\circ} \mathrm{C}$.

5-Chloro-2-methylthio-4-phenylpyrimidine (11b). ${ }^{3}$ The crude product was purified by flash chromatography (toluene); m.p. $70^{\circ} \mathrm{C}$.

2-Chloro-4-( $\beta$-styryl)pyrimidine (12a). Dichloromethane was used for flash chromatography; m.p. $142^{\circ} \mathrm{C}$. Mol.wt: Found 216.0438. Calc. for $\mathrm{C}_{12} \mathrm{H}_{9} \mathrm{ClN}_{2}$ : 216.0454. ${ }^{1} \mathrm{H}$ NMR: $\delta 6.85$ and $7.81(\mathrm{CH}=\mathrm{CH}, 2 \times \mathrm{d}, J 16 \mathrm{~Hz}), 7.08(1 \mathrm{H}, \mathrm{d}, J 5$
$\mathrm{Hz}, \mathrm{H}-5), 7.1-7.6(\mathrm{Ph}), 8.39$ (1 H, d, J $5 \mathrm{~Hz}, \mathrm{H}-6)$. MS: 218/216 (17/53, M), 217/215 (37/100), 182 (2), 181 (19), 180 (4), 179 (15), 154 (18).

2-Chloro-4-phenylpyrimidine (12b). ${ }^{28}$ Toulene-EtOAc 98:2 was used for flash chromatography; m.p. $89^{\circ} \mathrm{C}$ (light petroleum). Anal. $\mathrm{C}_{10} \mathrm{H}_{7} \mathrm{ClN}_{2}: \mathrm{C}, \mathrm{H} .{ }^{1} \mathrm{H}$ NMR (300 MHz): $\delta$ 7.5 and $8.1(\mathrm{Ph}, 2 \times \mathrm{m}), 7.63(1 \mathrm{H}, \mathrm{d}, J 5.3 \mathrm{~Hz}, \mathrm{H}-5), 8.61$ (1 H, d, J 5.3 Hz, H-6). MS: 192/190 (35/93, M), 191 (17), 156 (9), 155 (43), 130 (12), 129 (100), 128 (28).

2,5-Dichloro-4-( $\beta$-styryl)pyrimidine (13a). Hexane-dichloromethane 1:1 was used for flash chromatography; m.p. $125^{\circ} \mathrm{C}$. Anal. $\mathrm{C}_{12} \mathrm{H}_{8} \mathrm{Cl}_{2} \mathrm{~N}_{2}$ : C, H. ${ }^{1} \mathrm{H}$ NMR: 7.1-7.8 (Ph, $\mathrm{CH}=), 8.13(\mathrm{CH}=$, d, $J 16 \mathrm{~Hz}), 8.44(1 \mathrm{H}, \mathrm{s}, \mathrm{H}-6)$. MS: 254/252/250 (9/64/100, M), 253/251/249 (17/75/97), 218 (3), 217 (22), 216 (12), 215 (77), 214 (6).

2,5-Dichloro-4-phenylpyrimidine (13b). Toluene-hexane 1:1 was used for flash chromatography; m.p. $71^{\circ} \mathrm{C}$. Anal. $\mathrm{C}_{10} \mathrm{H}_{6} \mathrm{Cl}_{2} \mathrm{~N}_{2}: \mathrm{C}, \mathrm{H} .{ }^{1} \mathrm{H}$ NMR $(300 \mathrm{MHz}): \delta 7.5$ and $7.9(\mathrm{Ph}$, $2 \times \mathrm{m}), 8.62(\mathrm{H}-6) .{ }^{13} \mathrm{C}$ NMR: $\delta 128.2$ and $129.3\left(\mathrm{C}_{o}\right.$ and $\left.\mathrm{C}_{m}\right), 130.9\left(\mathrm{C}_{p}\right), 127.5$ and $134.1\left(\mathrm{C}-5\right.$ and $\left.\mathrm{C}_{\mathrm{i}}\right), 158.7$ and 164.8 (C-4 and C-2), 159.6 (C-6). MS: 228/226/224 (6/40/66, $M), 227$ (6), 225 (10), 192 (6), 191 (42), 190 (15), 189 (100), 163 (22).

5-Bromo-2-chloro-4-( $\beta$-styryl)pyrimidine (14). Hexane-dichloromethane $3: 2$ was used for flash chromatography; m.p. $117^{\circ} \mathrm{C}$. Anal. $\mathrm{C}_{12} \mathrm{H}_{8} \mathrm{BrClN}_{2}$ : C, H. ${ }^{1} \mathrm{H}$ NMR: $7.0-7.8$ $(\mathrm{Ph}, \mathrm{CH}=\mathrm{CH}), 8.14(\mathrm{CH}=\mathrm{CH}, \mathrm{d}, J 16 \mathrm{~Hz}), 8.56(1 \mathrm{H}, \mathrm{s}$, H-6). MS: 298/296/294 (23/100/74, M), 297 (32), 295 (95), 293 (64), 261 (5), 259 (9), 217 (28), 215 (92).

5-Bromo-2-methylthio-4-( $\beta$-styryl)pyrimidine (15). Toluene-hexane 9:1 was used for flash chromatography; m.p. $93^{\circ} \mathrm{C}$ (EtOH). Mol.wt: Found 305.9836. Calc. for $\mathrm{C}_{13} \mathrm{H}_{11} \mathrm{BrN}_{2} \mathrm{~S}: 305.9827 .{ }^{1} \mathrm{H}$ NMR: $2.58(\mathrm{MeS}), 7.1-7.7(\mathrm{Ph}$, $\mathrm{CH}=\mathrm{CH}), 8.00(\mathrm{CH}=\mathrm{CH}, \mathrm{d}, J 16 \mathrm{~Hz}), 8.42(1 \mathrm{H}, \mathrm{s}, \mathrm{H}-6)$, MS: 308/306 (97/100, M), $307(34), 305(20), 293$ (4), 291 (5), 275 (8), 273 (12), 261 (16), 259 (17), 235 (20), 233 (23).

2-Phenyl-4-( $\beta$-styryl)pyrimidine (20a). ${ }^{29}$ Hexane-dichloromethane 2:5 was used for flash chromatography; m.p. $109^{\circ} \mathrm{C}$. Anal. $\mathrm{C}_{18} \mathrm{H}_{14} \mathrm{~N}_{2}: \mathrm{C}, \mathrm{H} .{ }^{1} \mathrm{H}$ NMR $(300 \mathrm{MHz}): \delta 7.08$ and $7.97(\mathrm{CH}=\mathrm{CH}, 2 \times \mathrm{d}, J 16.2 \mathrm{~Hz}), 7.10(\mathrm{H}-5, \mathrm{~d}, J 5.0$ $\mathrm{Hz}), 8.69(\mathrm{H}-6, \mathrm{~d}, J 5.2 \mathrm{~Hz}), 7.3-7.7\left(8 \mathrm{H}, \mathrm{Ph}\right.$ and $\left.\mathrm{C}_{6} \mathrm{H}_{3}\right)$, 8.5-8.6 $\left(2 \mathrm{H}, \mathrm{C}_{6} \mathrm{H}_{2}\right) .{ }^{13} \mathrm{C}$ NMR: $\delta 116.2(\mathrm{C}-5), 126.1,129.1$ and $130.4\left(\mathrm{CH}=, 2 \times \mathrm{C}_{p}\right), 127.5,128.1,128.3$ and 128.7 (2 $\times \mathrm{C}_{o}$ and $\left.2 \times \mathrm{C}_{m}\right), 135.7$ and $137.8\left(2 \times \mathrm{C}_{\mathrm{i}}\right), 136.8(\mathrm{CH}=)$, 157.5 (C-6), 162.2 and 164.2 (C-4 and C-2). MS: 258 (52, M) 257 (100), 231 (1), 230 (5), 181 (2), 179 (3), 156 (4), 155 (24), 154 (21).

4-Phenyl-2-( $\beta$-styryl)pyrimidine (20b). Dichloromethane was used for flash chromatography; m.p. $92^{\circ} \mathrm{C}$. Anal. 
$\mathrm{C}_{18} \mathrm{H}_{14} \mathrm{~N}_{2}: \mathrm{C}, \mathrm{H} .{ }^{1} \mathrm{H}$ NMR (300 MHz): $\delta 7.33$ and 8.10 $(\mathrm{CH}=\mathrm{CH}, 2 \times \mathrm{d}, J 16.1 \mathrm{~Hz}), 7.45(\mathrm{H}-5, \mathrm{~d}, J 5.1 \mathrm{~Hz}), 8.69$ (H-6, d, J 5.2 Hz), 7.3-7.7 (8 H, Ph and $\left.\mathrm{C}_{6} \mathrm{H}_{3}\right), 8.1-8.2(2$ $\left.\mathrm{H}, \mathrm{C}_{6} \mathrm{H}_{2}\right) .{ }^{13} \mathrm{C}$ NMR: $\delta 114.0(\mathrm{C}-5), 127.0,127.5,128.6$ and $128.7\left(2 \times \mathrm{C}_{o}\right.$ and $\left.2 \times \mathrm{C}_{m}\right), 127.8,128.8$ and $130.7(\mathrm{CH}=$ and $\left.2 \times \mathrm{C}_{p}\right), 136.0$ and $136.8\left(2 \times \mathrm{C}_{\mathrm{i}}\right), 137.8(\mathrm{CH}=), 157.3$ (C-6), 163.7 and 164.7 (C-4 and C-2). MS: $258(33, M), 257$ (100), 256 (2), 230 (1), 181 (2), 156 (1), 155 (5), 128 (15).

2-Chloro-5-phenyl-4-( $\beta$-styryl)pyrimidine (21). Toluene was used for flash chromatography; m.p. $120^{\circ} \mathrm{C}(\mathrm{EtOH})$. Anal. $\mathrm{C}_{18} \mathrm{H}_{13} \mathrm{ClN}_{2}$ : C, H. ${ }^{1} \mathrm{H}$ NMR $(300 \mathrm{MHz}): \delta 7.06$ and 8.13 $(\mathrm{CH}=\mathrm{CH}, 2 \times \mathrm{d}, J 15.6 \mathrm{~Hz}), 7.3-7.6(2 \times \mathrm{Ph}), 8.47(\mathrm{H}-6)$. ${ }^{13} \mathrm{C}$ NMR: $\delta 121.5(\mathrm{CH}=), 127.9,128.7$ (br), 128.9 and $129.4\left(2 \times \mathrm{C}_{o}\right.$ and $\left.2 \times \mathrm{C}_{m}\right), 128.7(\mathrm{br})$ and $129.6\left(2 \times \mathrm{C}_{p}\right)$, $131.4,134.0$ and $135.4\left(2 \times \mathrm{C}_{\mathrm{i}}\right.$ and $\left.\mathrm{C}-5\right), 139.8(\mathrm{CH}=)$, 159.7 and 161.9 (C-4 and C-2), 160.0 (C-6). MS: 294/292 (28/85, M), 293 (46), 291 (100), 257 (9), 256 (7), 255 (25), 230 (15), 229 (6), 228 (10), 227 (18), 217 (31), 216 (16), 215 (95).

5-Phenyl-4-( $\beta$-styryl)-2-(2-thienyl)pyrimidine (22). Toluene was used for flash chromatography; m.p. $128^{\circ} \mathrm{C}(\mathrm{EtOH})$. Anal. $\mathrm{C}_{22} \mathrm{H}_{16} \mathrm{~N}_{2} \mathrm{~S}$ : C, H. ${ }^{1} \mathrm{H}$ NMR (300 MHz): $\delta 7.15$ and $8.19(\mathrm{CH}=\mathrm{CH}, 2 \times \mathrm{d}, J 15.6 \mathrm{~Hz}), 7.16(1 \mathrm{H}, \mathrm{dd}, J 5.0,3.7$ $\mathrm{Hz}), 7.2-7.6(11 \mathrm{H}, 2 \times \mathrm{Ph}$ and $1 \mathrm{H}$ thienyl $), 8.12(1 \mathrm{H}$, dd, $J 3.7,1.2 \mathrm{~Hz}$ ), 8.58 (H-6). ${ }^{13} \mathrm{C}$ NMR: $\delta$ 123.0, 128.0, 128.1, 128.6 and $129.0\left(\mathrm{CH}=, 2 \times \mathrm{C}_{p}\right.$ and $3 \times \mathrm{CH}$ thienyl $), 127.7$, 128.5, 128.7 and $129.4\left(2 \times \mathrm{C}_{o}\right.$ and $\left.2 \times \mathrm{C}_{m}\right), 130.1,135.4$, 136.0 and $143.5\left(2 \times \mathrm{C}_{\mathrm{i}}, \mathrm{C}-5\right.$ and $\mathrm{C}_{\mathrm{i}}$ thienyl $), 137.8(\mathrm{CH}=)$, 158.0 (C-6), 158.6 and 159.5 (C-4 and C-2). MS: 340 (77, M), 339 (81), 265 (6), 264 (19), 263 (100), 255 (5), 231 (9), 230 (12).

5-Chloro-4-(a-ethoxyvinyl)-2-methylthiopyrimidine (23a). Hexane-dichloromethane 2:1 was used for flash chromatography; m.p. $44^{\circ} \mathrm{C}$ (sublimation at $50^{\circ} \mathrm{C} / 0.001 \mathrm{mmHg}$ ). 'H NMR: $\delta 1.39$ and 3.93 (EtO), 2.56 (MeS), 4.55 and 4.87 $\left(\mathrm{C}=\mathrm{CH}_{2}, 2 \times \mathrm{d}, J 3 \mathrm{~Hz}\right), 8.46(1 \mathrm{H}, \mathrm{s} \mathrm{H}-6)$. MS: $232 / 230$ (4/13, M), 213 (2), 217 (2), 215 (7), 203 (4), 202 (2), 201 (12), 188 (37), 186 (100).

2-( $\alpha$-Ethoxyvinyl)-5-methylpyrimidine (23b). Dichloromethane-EtOAc 15:1 was used for flash chromatography; m.p. $80^{\circ} \mathrm{C}$ (sublimation at $50^{\circ} \mathrm{C} / 0.01 \mathrm{mmHg}$ ). Anal. $\mathrm{C}_{9} \mathrm{H}_{12} \mathrm{~N}_{2} \mathrm{O}: \mathrm{C}, \mathrm{H} .{ }^{1} \mathrm{H}$ NMR (300 MHz): $\delta 1.50(3 \mathrm{H}, \mathrm{t}, J 7.0$ $\mathrm{Hz}), 2.32(\mathrm{Me}), 4.05(2 \mathrm{H}, \mathrm{q}, J 7.0 \mathrm{~Hz}), 4.59$ and 5.62 $\left(\mathrm{C}=\mathrm{CH}_{2}, 2 \times \mathrm{d}, J 2.1 \mathrm{~Hz}\right), 8.59(\mathrm{H}-4,6) .{ }^{13} \mathrm{C}$ NMR: $\delta 14.2$ (Me), 15.3 (5-Me), $63.9\left(\mathrm{CH}_{2}-\mathrm{O}\right), 88.9\left(\mathrm{CH}_{2}=\right), 129.0$ (C-5), $156.9(\mathrm{C}-4,6), 157.3$ and 159.4 (=C-OEt and C-2). MS: $164(26, M), 149(23), 121(11), 120(82), 119$ (28), 108 (18), 95 (9), 94 (100).

2-Chloro-5-(a-ethoxyvinyl)pyrimidine (23c). Hexane-dichloromethane $3: 4$ was used for flash chromatography; m.p. $86^{\circ} \mathrm{C}(\mathrm{EtOH})$ : Anal. $\mathrm{C}_{8} \mathrm{H}_{9} \mathrm{ClN}_{2} \mathrm{O}: \mathrm{C}, \mathrm{H} .{ }^{1} \mathrm{H}$ NMR: $\delta$
1.43 and 3.94 (EtO), 4.38 and $4.72\left(\mathrm{C}=\mathrm{CH}_{2}, 2 \times \mathrm{d}, J 4\right.$ $\mathrm{Hz}), 8.83$ (2 H, s, H-4,6). MS: 186/184 (7/23, M), 185 (6), 183 (7), 158 (16), 156 (45), 143 (32), 142 (15), 141 (100), $140(23)$.

5-Chloro-2-methylsulfonyl-4-( $\beta$-styryl)pyrimidine (16). A solution of 5-chloro-2-methylthio-4-( $\beta$-styryl)pyrimidine $(1.09 \mathrm{~g}, 4.15 \mathrm{mmol})$ and $m$-chloroperbenzoic acid $(2.17 \mathrm{~g}$, $12.46 \mathrm{mmol})$ in dichloromethane $(100 \mathrm{ml})$ was left to stand at ambient temperature for $3 \mathrm{~h}$ before it was shaken consecutively with saturated aqueous sodium sulfite, saturated aqueous sodium hydrogen carbonate and water. The dried $\left(\mathrm{MgSO}_{4}\right)$ solution was evaporated and the residual material was crystallized from 2-propanol; yield $0.97 \mathrm{~g} \mathrm{(80 \% ),} \mathrm{m.p.}$ $131^{\circ} \mathrm{C}$. Anal. $\mathrm{C}_{13} \mathrm{H}_{11} \mathrm{ClN}_{2} \mathrm{O}_{2} \mathrm{~S}$ : C, H. ${ }^{1} \mathrm{H}$ NMR: $\delta 3.38$ $\left(\mathrm{MeSO}_{2}\right), 7.2-7.8(\mathrm{Ph}, \mathrm{CH}=\mathrm{CH}), 8.23(\mathrm{CH}=\mathrm{CH}, \mathrm{d}, J 16$ Hz), 8.68 (1 H, s, H-6). MS: 296/294 (3/9, M), 293 (3), 231 (4), 217 (15), 216 (38), 215 (47), 214 (100), 187 (17).

5-Bromo-2-methylsulfonyl-4-( $\beta$-styryl)pyrimidine

(17). Compound 17 was prepared as above from 5-bromo-2methylthio-4-( $\beta$-styryl)pyrimidine. The reaction was run for $17 \mathrm{~h}$ at ambient temperature; yield $75 \%$, m.p. $159^{\circ} \mathrm{C}$ (2-propanol). Anal. $\mathrm{C}_{13} \mathrm{H}_{11} \mathrm{BrN}_{2} \mathrm{O}_{2} \mathrm{~S}$ : C, H. ${ }^{1} \mathrm{H}$ NMR: $\delta$ $3.39\left(\mathrm{MeSO}_{2}\right), 7.3-7.9(\mathrm{Ph}, \mathrm{CH}=\mathrm{CH}), 8.32(\mathrm{CH}=\mathrm{CH}, \mathrm{d}, J$ $16 \mathrm{~Hz}), 8.90$ (1 H, s, H-6). MS: 340/338 (4/3, M), 339 (2) 337 (2), 294 (2), 277 (2), 275 (2), 261 (22), 260 (52), 259 (25), 258 (53), 179 (100).

5-Chloro-4-( $\beta$-styryl)-2(1H)-pyrimidinone (18): Method $A$. Sodium hydroxide $(1 \mathrm{M}, 300 \mathrm{ml})$ was added to a solution of 5-chloro-2-methylsulfonyl-4-( $\beta$-styryl)pyrimidine (3.03 g, $10.28 \mathrm{mmol})$ in dioxane $(250 \mathrm{ml})$, and the mixture was stirred at ambient temperature for $50 \mathrm{~h}$, after which time it was cooled to $0^{\circ} \mathrm{C}$ and neutralized with hydrochloric acid. The precipitated product was dried and recrystallized from ethanol; yield $2.04 \mathrm{~g}(85 \%)$, m.p. $246^{\circ} \mathrm{C}$. Anal $\mathrm{C}_{12} \mathrm{H}_{9} \mathrm{ClN}_{2} \mathrm{O}: \mathrm{C}, \mathrm{H} .{ }^{1} \mathrm{H}$ NMR [ $\left.\left(\mathrm{CD}_{3}\right)_{2} \mathrm{SO}\right]: \delta 7.33$ and 7.98 $(\mathrm{CH}=\mathrm{CH}, 2 \times \mathrm{d}, \mathrm{J} 16 \mathrm{~Hz}), 7.3-7.9(\mathrm{Ph}), 8.40(1 \mathrm{H}, \mathrm{s}, \mathrm{H}-6)$. MS: 234/232 (23/71, M), 233/231 (42/100), 197 (19), 169 (14), 168 (13), 155 (11), 154 (23).

Method $B$. Sodium hydroxide $(1 \mathrm{M}, 15 \mathrm{ml})$ was added to a solution of 2,5 -dichloro-4-( $\beta$-styryl)pyrimidine $(0.50 \mathrm{~g}$, $1.99 \mathrm{mmol})$ in dioxane $(5 \mathrm{ml})$. The mixture was heated under reflux for $2 \mathrm{~h}$, cooled to $0^{\circ} \mathrm{C}$, and worked up as above; yield $0.12 \mathrm{~g}(26 \%)$.

5-Bromo-2-methoxy-4-( $\beta$-styryl)pyrimidine (19): Method $A$. Sodium methoxide $(60 \mathrm{mg}, 1.11 \mathrm{mmol})$ was added to a solution of 5-bromo-2-methylsulfonyl-4-( $\beta$-styryl)pyrimidine $(250 \mathrm{mg}, 0.74 \mathrm{mmol})$ in methanol $(5 \mathrm{ml})$. The mixture was heated under reflux for $2 \mathrm{~h}$, then cooled and poured into aqueous ammonium chloride. The mixture was extracted with diethyl ether, the dried $\left(\mathrm{MgSO}_{4}\right)$ ether solution was evaporated, and the residual material was crystallized from methanol; yield $211 \mathrm{mg}(98 \%)$, m.p. $73^{\circ} \mathrm{C}$. 
Anal. $\mathrm{C}_{13} \mathrm{H}_{11} \mathrm{BrN}_{2} \mathrm{O}: \mathrm{C}, \mathrm{H} .{ }^{1} \mathrm{H}$ NMR: $\delta 4.06(\mathrm{OMe})$, 7.2-7.8 $(\mathrm{Ph}, \mathrm{CH}=\mathrm{C}), 8.15(\mathrm{CH}=\mathrm{CH}, \mathrm{d}, J 16 \mathrm{~Hz}), 8.55(1$ H, s, H-6). MS: 292/290 (71/75, M), 291/289 (100/94), 277/ 275 (9/9), 211 (50), 196 (41), 195 (17), 179 (13).

Method B. Sodium methoxide $(0.22 \mathrm{~g}, 4.06 \mathrm{mmol})$ was added to a solution of 5-bromo-2-chloro-4-( $\beta$-styryl)pyrimidine $(0.80 \mathrm{~g}, 2.71 \mathrm{mmol})$ in methanol $(15 \mathrm{ml})$. The mixture was heated under reflux for $2 \mathrm{~h}$ and worked up as above; yield $0.74 \mathrm{~g}(94 \%)$.

4-Acetyl-5-chloro-2-methylthiopyrimidine (24a). 5Chloro-4-( $\alpha$-ethoxyvinyl)-2-methylthiopyrimidine $(222 \mathrm{mg}$, $0.96 \mathrm{mmol}$ ) was added to acetone-1 $\mathrm{M} \mathrm{HCl} \mathrm{4:1} \mathrm{(5} \mathrm{ml)} \mathrm{and}$ the mixture was stirred at ambient temperature for $18 \mathrm{~h}$ and heated under reflux for $1 \mathrm{~h}$. Water $(10 \mathrm{ml})$ was then added and the mixture was extracted with diethyl ether $(3 \times 10$ $\mathrm{ml})$. The ether extracts were washed with saturated aqueous sodium hydrogen carbonate, dried $\left(\mathrm{MgSO}_{4}\right)$, and evaporated, and the product was isolated by sublimation at $40^{\circ} \mathrm{C} / 0.01 \mathrm{mmHg}$; yield $179 \mathrm{mg}(92 \%)$, m.p. $46^{\circ} \mathrm{C}$. Anal. $\mathrm{C}_{7} \mathrm{H}_{7} \mathrm{ClN}_{2} \mathrm{OS}: \mathrm{C}, \mathrm{H} .{ }^{1} \mathrm{H}$ NMR (300 MHz): $\delta 2.59(\mathrm{MeS})$, 2.66 (MeCO), 8.61 (H-6). ${ }^{13} \mathrm{C}$ NMR: 814.4 (MeS), 27.6 $(\mathrm{MeCO}), 122.3$ (C-5), 156.2 (C-4), 159.2 (C-6), 170.7 (C-2), 197.3 (C=O). MS: 204/202 (9/27, M), 174 (4), 173 (4), 161 (8), 160 (11), 159 (14), 149 (17), 43 (100).

2-Acetyl-5-methylpyrimidine (24b). Compound 24b was prepared as above from 2-( $\alpha$-ethoxyvinyl)-5-methylpyrimidine. The reaction was left at ambient temperature for 18 $\mathrm{h}$, water $(10 \mathrm{ml})$ was then added and the mixture was extracted with chloroform $(3 \times 10 \mathrm{ml})$. The chloroform extracts were dried $\left(\mathrm{MgSO}_{4}\right)$ and evaporated and the product was isolated by sublimation at $40^{\circ} \mathrm{C} / 0.01 \mathrm{mmHg}$. Yield $80 \%$; m.p. $49^{\circ} \mathrm{C}$. Anal. $\mathrm{C}_{7} \mathrm{H}_{8} \mathrm{~N}_{2} \mathrm{O}$ : C, H. ${ }^{1} \mathrm{H}$ NMR $(300$ $\mathrm{MHz}): \delta 2.46(\mathrm{Me}), 2.77(\mathrm{MeCO}), 8.77(\mathrm{H}-4,6) .{ }^{13} \mathrm{C}$ NMR: $\delta 15.3(\mathrm{Me}), 26.2$ (MeCO), 132.7 (C-5), 157.0 (C-4. 6), 157,6 (C-2), $197.0(\mathrm{C}=\mathrm{O})$. MS: $136(81, M), 121(8), 108$ (24), 95 (7), 94 (100), 93 (66), 67 (43).

5-Acetyl-2-chloropyrimidine (24c). Compound $24 \mathrm{c}$ was prepared as above from 2 -chloro-5-( $\alpha$-ethoxyvinyl)pyrimidine. The reaction was left at ambient temperature for $18 \mathrm{~h}$. Yield $80 \%$; m.p. $92^{\circ} \mathrm{C}$ (sublimed at $25^{\circ} \mathrm{C} / 0.01 \mathrm{mmHg}$ ). Anal. $\mathrm{C}_{6} \mathrm{H}_{5} \mathrm{ClN}_{2} \mathrm{O}: \mathrm{C}, \mathrm{H} .{ }^{1} \mathrm{H}$ NMR: $\delta 2.67(\mathrm{MeCO}), 9.10(2$ H, s, H-4,6). MS: 158/156 (7/29, M), 149 (7), 145 (5), 143 (29), 141 (100), $136(30), 113$ (17), 94 (58), 93 (43).

\section{References}

1. Rise, F. and Undheim, K. J. Chem Soc., Perkin Trans. I (1985) 1997.

2. Solberg, J. and Undheim, K. Acta Chem. Scand., Ser. B 40 (1986) 381

3. Solberg, J. and Undheim, K. Acta Chem. Scand., Ser. B 41 (1987) 712 .

4. (a) Sandosham, J., Benneche, T., Møller, B. S. and Undheim, K. Acta Chem. Scand., Ser. B 42 (1988) 455; (b) Arukwe, J., Benneche, T. and Undheim, K. J. Chem. Soc., Perkin Trans. 1. In press.

5. Brown, D. J. The Pyrimidines, The Chemistry of Heterocyclic Compounds. Interscience (1962), Supplement 1 (1970). Supplement 2 (1985).

6. Stille, J. K. Angew. Chem., Int. Ed. Engl. 25 (1986) 508.

7. McKean, D. R., Parrinello, G., Renaldo, A. F. and Stille, J. K. J. Org. Chem. 52 (1987) 422.

8. Stille, J. K. Pure Appl. Chem. 57 (1985) 1771.

9. Edo, K., Sakamto, T. and Yamanaka, H. Chem. Pharm. Bull. Jpn. 26 (1978) 3843.

10. Gronowitz, S., Hörnfeldt, A.-B., Kristjansson, V. and Musil. T. Chem. Scr. 26 (1986) 305.

11. Saihi, M. L. and Pereyre, M. Bull. Soc. Chim. Fr. 11-12 (1977) 1251.

12. Baily, T. R. Tetrahedron Lett. 27 (1986) 4407.

13. Wardell, J. L. and Ahmed. S. J. Organomet. Chem. 78 (1974) 395.

14. Brown, D. J. and Waring, P. Aust. J.Chem. 26 (1973) 443.

15. English, J. P., Clark, J. H., Shepherd, R. G., Marson, H. W. Krapcho, J. and Roblin, R. O., Jr. J. Am. Chem. Soc. 68 (1946) 1039.

16. Brown, D. J. and Lyall, J. M. Aust. J. Chem. 17 (1964) 794.

17. Benneche, T., Gacek, M. and Undheim, K. European Pat. Appl. 33,195. (1981); Chem. Abstr. 95 (1981) 187298e.

18. (a) Matsukawa, T. and Ohta, B. J. Pharm. Soc. Jpn. 69 (1949) 491; Chem. Abstr. 44 (1950) 3456d; (b) Wheeler, H. L. and Bristol, H. S. Am. Chem. J. (1905) 437.

19. Hurst, D. T. and Johnson, M. Heterocycles 23 (1985) 611.

20. Chesterfield, J., McOmie, J. F. W. and Sayer, E. R. J. Chem. Soc. (1955) 3478.

21. Whittaker, N. J. Chem. Soc. (1953) 1646.

22. Johnson, T. B. and Joyce, A. W. J. Am. Chem. Soc. 38 (1916) 1557.

23. Soderquist, J. A. and Hsu, G.J.-H. Organometallics (1982) 830.

24. Holland, A. Chem. Ind. (London) (1954) 786.

25. Burdeska, K., Fuhrer, H., Kabas, G. and Siegrist, A. E. Helv. Chim. Acta 64 (1981) 113.

26. Brown, D. J. and Lee, T.-C. J. Chem. Soc. C (1970) 214.

27. Kroon, A. P., van der Plas, H. C. and van Garderen, G. Recl. Trav. Chim. Pays-Bas 93 (1974) 325.

28. Matsukawa, T. and Ohta, B. J. Pharm. Soc. Jpn. 70 (1950) 134; Chem. Abstr. 44 (1950) 5886d.

29. Heyes, T. D. and Roberts, J. C. J. Chem. Soc. (1951) 328.

Received June 29, 1988. 